\title{
The use of weightbearing radiographs to assess the stability of supination-external rotation fractures of the ankle
}

\author{
Martin Weber · Helge Burmeister • \\ Gerhard Flueckiger · Fabian G. Krause
}

Received: 2 June 2009/Published online: 16 January 2010

(C) Springer-Verlag 2010

\begin{abstract}
Background Isolated lateral malleolar fractures usually result from a supination-external rotation (SER) injury and may include a deltoid ligament rupture. The necessity of operative treatment is based on the recognition of a relevant medial soft-tissue disruption. Currently used tests to assess ankle stability include manual stress radiographs and gravity stress radiographs, but seem to overestimate the need for fracture fixation.

Methods We investigated the use of weightbearing radiographs to distinguish stable and unstable isolated lateral malleolar fractures induced by the SER mechanism in 57 patients. Patients with stable fractures (SER type II according to the Lauge-Hansen classification) were treated non-operatively with varying external support. Forty-seven patients were evaluated by questionnaire and AOFAS ankle-hindfoot score. Follow-up was 18-120 months (mean 62).

Results Fifty-one of fifty-seven (90\%) patients were found to have stable fractures (SER type II) and were treated nonoperatively. The AOFAS score was 96.1 points on average (range 85-100) at latest follow-up. Four patients reported minor complaints. A "moderate" correlation of risk factors
\end{abstract}

M. Weber $(\bowtie) \cdot$ F. G. Krause

Department of Orthopaedic Surgery, University of Bern, Inselspital, 3010 Bern, Switzerland

e-mail: martin.weber@insel.ch

H. Burmeister

Department of Orthopaedic Surgery,

Kantonsspital, Schwyz, Switzerland

G. Flueckiger

Department of Orthopaedic Surgery,

Zieglerspital, Berne, Switzerland (i.e. smoking) to delayed bone healing was found while the correlation of varying external support (i.e. bandage, cast) to the AOFAS score and delayed bone healing was "poor". Conclusion The use of weightbearing radiographs is an easy, pain-free, safe and reliable method to exclude the need for operative treatment, with excellent clinical outcome in the majority of the patients seen at latest follow-up. The delay of 3-10 days until the decision about surgical treatment is well accepted by the patients.

Keywords Ankle · Fracture - Stability assessment . Radiographs $\cdot$ Non-operative treatment

\section{Introduction}

Isolated lateral malleolar fractures with competent deltoid ligament [supination external rotation fracture type II (SER-II) according to the Lauge-Hansen classification or AO-B1 according to the Mueller classification] are considered stable and are treated non-operatively with excellent results [1-4]. Fractures with clear lateral subluxation of the talus as seen on the initial non-weightbearing radiographs (SER-IV or AO-B2) are considered unstable and are thought to benefit from operative reduction and internal fixation. For patients with radiographically stable ankles, but clinical symptoms suggesting medial ligament injury, radiographs with either manual or gravity stress have been proposed to detect deltoid ligament incompetence [5-9].

Patients with radiological "medial clear space" of $4 \mathrm{~mm}$ or more, and an increase of $1 \mathrm{~mm}$ or more compared to the "superior clear space" are advised to have surgical stabilization [9]. This recommendation is based on the assumption that these values reflect the tidemark beyond 
which operative treatment is superior to non-operative treatment. However, this is not substantiated by the literature and a satisfactory outcome with non-operative treatment can be achieved even in fractures with positive stress radiographs but absent medial tenderness [5].

In this study, weightbearing radiographs were used to identify stable and unstable lateral malleolar fractures with SER mechanism since the weightbearing condition was thought to be more representative of the normal use of the ankle joint than non-weightbearing stress radiographs. The objective of the study was to assess the use of weightbearing radiographs with regard to the outcome of the patients who had been considered appropriate for nonoperative treatment.

\section{Patients and methods}

In this retrospective study, 78 patients (40 men and 38 women, age 16-87 years, mean 46 years) with closed isolated lateral malleolar fractures of the SER type according to Lauge-Hansen were seen at our institution from February 1997 to December 2006 [1]. Patients with a history of previous hindfoot trauma, pre-existing ligamentous instability or hindfoot pain, clinical signs of foot deformity, and radiographic signs of pre-existing ankle/ hindfoot arthritis or ankle/hindfoot malalignment were excluded. Twenty patients showed clear lateral subluxation of the talus (lateral edge of talar dome surpassing lateral edge of tibial plafond and widening of the medial clear space) on the initial non-weightbearing anteroposterior radiographs, and were advised to have surgical stabilization. Fifty-eight patients had no radiographic signs of instability on the initial non-weightbearing anteroposterior radiographs, and were included in the study group.

The patients of the study group were treated with varying external support and were kept partially weightbearing awaiting weightbearing radiographs 3-7 days later after the pain had mostly resolved (Fig. 1). Two patients did not return and were operated elsewhere, with no further examinations proving talar instability. Fifty-six patients were seen at 3-10 days (mean 6.7 days) after the injury, and weightbearing radiographs were taken (full bodyweight equally distributed on both legs, anteroposterior ankle radiograph in $10^{\circ}$ of internal rotation). Fractures were considered unstable (SER-IV, Fig. 2) when the medial clear space was $>4 \mathrm{~mm}$ and/or $>1 \mathrm{~mm}$ wider than the superior clear space, and when the lateral edge of the talar dome was surpassing the lateral edge of tibial plafond). For these patients, surgical stabilization was recommended.

The presence of a smoking history within last 5 years (14 patients), diabetic polyneuropathy and angiopathy (10),
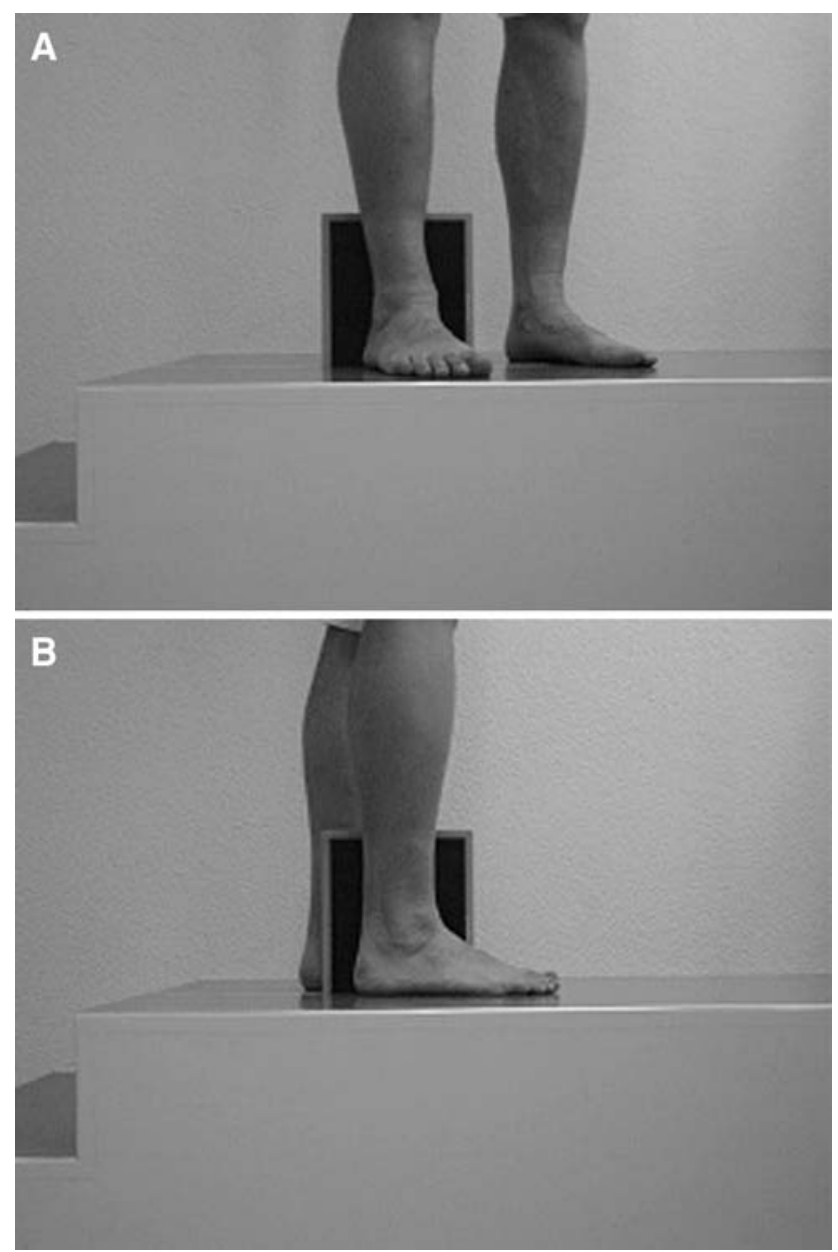

Fig. 1 Set-up for the weightbearing radiographs. The patient is standing on both legs as comfortably as possible, with the weight equally distributed. For the anteroposterior view (a), the foot is internally rotated $10^{\circ}$ by the patient turning the entire body, not only the leg. The lateral view (b) is taken lateromedially, using the same set-up

and obesity (4) were considered as risk factors and comorbidities potentially influencing bone healing [10]. Correlations of the risk factors and comorbidities to AOFAS score and complications were calculated.

For the patients in the study group with stable SER-II ankle fractures, initial external support for treatment ranged from bandages, orthotics, softcast-boots, and below-knee cast. Because SER-II fractures were considered stable, the patient's symptoms (pain and swelling) were the only indication for a below-knee cast. The cast was usually removed after $2-4$ weeks ( 3.1 weeks on average). Radiographs were taken at 6-week follow-up. If symptoms had not resolved at this time, and if there was still doubt about solid bony union and stability of the talus, the patients were seen again for another follow-up with new radiographs 4 weeks later. The patients were invited for the 1-year follow-up or earlier if they had any problems. 

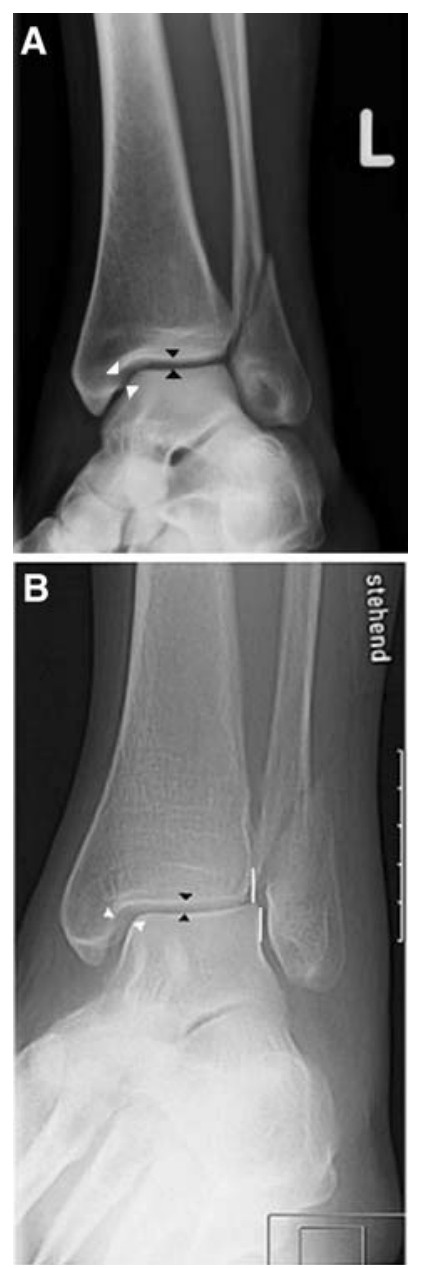

Fig. 2 Anteroposterior radiograph of an unstable fracture, nonweightbearing (a) and weightbearing (b). A medial clear space (white arrowheads) more than $4 \mathrm{~mm}$ in width and/or $1 \mathrm{~mm}$ wider than the superior clear space (black arrowheads) was considered as radiographic instability. The medial clear space is $4.7 \mathrm{~mm}$ on the weightbearing film indicating instability, the superior clear space is not widened but the lateral edge of talar dome is surpassing the lateral edge of tibial plafond (white lines)

At latest follow-up, the patients were contacted by phone. They were asked for any adverse events (operations) regarding their ankle since the fracture, for activity-related discomfort, and for impaired function of the hindfoot as opposed to prior to the injury. The AOFAS ankle-hindfoot score was used to illustrate the results and the correlation of type of non-operative treatment (bandages, orthotics, softcast-boots, and below-knee cast) to functional outcome as measured by the AOFAS score and the number of complications were calculated [11]. No radiographs were taken at latest follow-up.

Correlations were analyzed using the simple linear regression model. Spearman's rank correlation coefficient $r$ was interpreted as "poor" below 0.30 , "fair" from 0.31 to 0.50 , "moderate" from 0.51 to 0.60 , "moderately strong" from 0.61 to 0.80 , and "very strong" at or above 0.81 [11].
$P$ values less than 0.05 were considered significant. Statistical calculations were performed with BioStat $^{\circledR}$ software (AnalystSoft, Vancouver, Canada).

\section{Results}

Evaluation of the radiographs revealed lateral talar subluxation (medial clear space $>4 \mathrm{~mm}$ and/or $>1 \mathrm{~mm}$ than superior clear space, lateral edge of talar dome surpassing lateral edge of tibial plafond) in five patients. These fractures were considered unstable (SER-IV, Fig. 2), and surgical stabilization was recommended. Fifty-one patients had no signs of radiographic instability and remained in the study group (SER-II, Fig. 3). For the patients in the study group with stable SER-II ankle fractures initial external support for treatment ranged from bandages (11 patients), orthotics (4), softcast-boots (7), and below-knee cast (25).

Of the 51 patients with stable injury, 47 were reached for latest follow-up and questioned by phone. The followup period ranged from 18 to 120 months (mean 62). Four patients had died, but no ankle problems had been previously reported to the relatives. No patient had an operation for the ankle fracture or any other adverse event regarding the ankle joint in the interim. No patient reported major activity-related discomfort, or complained about an impaired function of the hindfoot as opposed to prior to the injury. The average AOFAS ankle-hindfoot score was calculated 96.1 points (range 85-100) (Table 1). The correlation of type of external support (bandages, orthotics, softcast-boots, and below-knee cast) and functional outcome as measured by the AOFAS score was "poor" $(r=0.28, p \geq 0.05)$.

During the initial phase of treatment, few complications were encountered. At 6 weeks follow-up, no callus was seen in six patients (of 42 with radiographs). Of those, two patients were smokers ( 2 of overall 14 smokers), two were diabetic (2/10), and one was obese (1/4, BMI 32).

At 3 months, two of these patients (1 smoker) still had not fully healed fractures, but were healed after 6 months without further treatment. One patient (no risk factor/ comorbidity) had neuropathic pain, while the fracture was fully healed. The ankle functioned well, and an MRI and the neurological assessment were normal. At 13 months, the pain was moderate, and the clinical and radiological examination was normal, specifically no instability or arthritis was found. The patient was subsequently lost to follow-up. One patient (no risk factor/comorbidity) developed a complex regional pain syndrome, which had healed completely at latest follow-up.

Correlation of the risk factors and comorbidities to AOFAS score and complications (delayed bone healing) 
Fig. 3 Anteroposterior view of a stable fracture, nonweightbearing (a) and weightbearing (b). On the weightbearing film, the medial clear space (white arrowheads) is below $4 \mathrm{~mm}(3.7 \mathrm{~mm})$, has the same width as the superior clear space (black arrowheads, and the lateral edge of talar dome is not surpassing the lateral edge of tibial plafond (white lines)
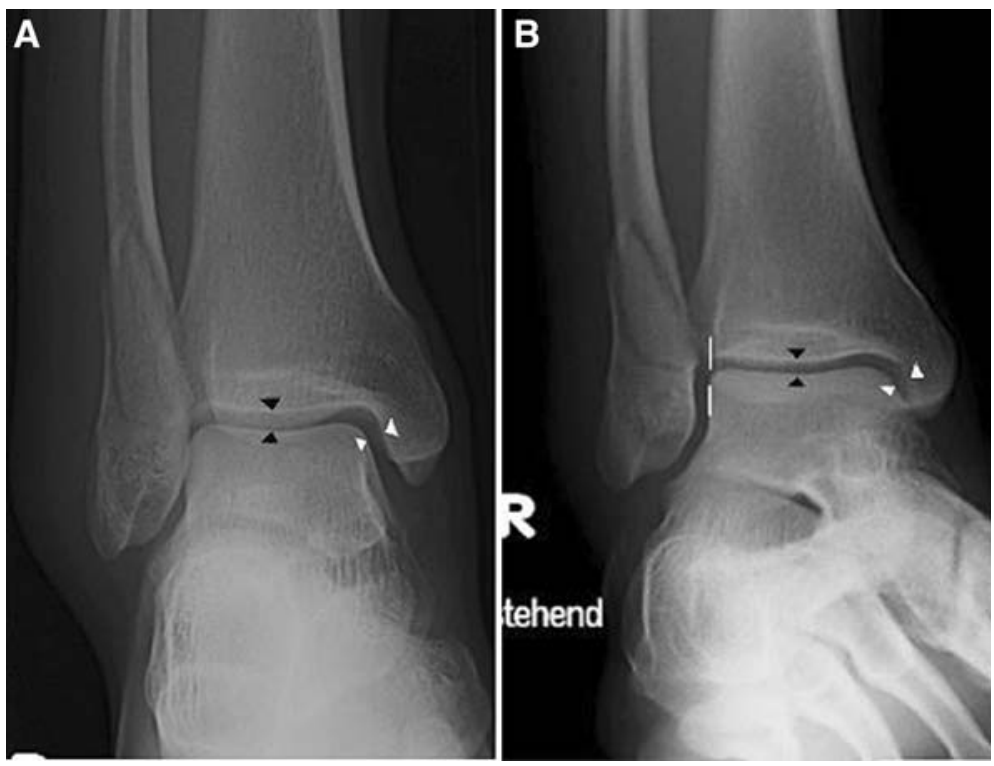

Table 1 Clinical results according to the AOFAS ankle-hindfoot score at latest follow-up (mean 63 months), by questionnaire

\begin{tabular}{lc}
\hline $\begin{array}{l}\text { AOFAS-score } \\
\text { (max. } 100 \text { points) }\end{array}$ & $\begin{array}{l}\text { Total of } \\
47 \text { patients }\end{array}$ \\
\hline Pain & 41 \\
None (40 points) & 6 \\
$\quad$ Mild, occasional (30 points) & \\
Function & 42 \\
$\quad$ Normal (50 points) & 3 \\
Exc. limitation of recreational & \\
$\quad$ activities ( 47 points) & 2 \\
Above + some difficulty on & uneven terrains (45 points) \\
Alignment & \\
Normal (10 points) & 47 \\
\hline
\end{tabular}

was calculated "moderate" $(r=0.58$ and $r=0.56$, $p \geq 0.05)$. The correlation of type of external support (bandages, orthotics, softcast-boots, and below-knee cast) and complications was "poor" $(r=0.25, p \geq 0.05)$.

\section{Discussion}

Recognizing a functionally relevant deltoid ligament injury is crucial when deciding about operative or non-operative treatment of an isolated lateral malleolar fracture. In situations where the instability is not clearly visible on the initial radiographs, stress radiographs are advised, since soft-tissue indicators (swelling, tenderness, ecchymosis) are not accurate predictors of instability [5, 7-9]. Recommendations for operative treatment of unstable injuries are made by most authors on the basis of the results presented by Yde and Kristensen in 1980, about SER fractures stage
IV [12]. In this study, the authors compared 60 operated patients of one center to 29 non-operated patients of the other center and concluded that operative treatment gave better results. However, only 25 of the operated patients, and eight of the non-operated patients had a deltoid ligament rupture. Furthermore, the number of patients having additional fractures of the posterior tibial margin is not known. Subgroup outcome analysis was not performed. It is therefore not admissible to make a conclusive statement about the superiority of operative treatment of the isolated lateral malleolar fracture with deltoid ligament rupture. Specifically, it is not known which amount of lateral talar displacement implies a relevant instability.

Good results after non-operative treatment in minor instability were seen by Egol et al. [13]. Since weightbearing radiographs represent a condition more closely representative of the actual clinical situation, this test was chosen to distinguish unstable from stable isolated lateral malleolar fractures, and to allow recommendation for operative and non-operative treatment. Only five of 57 patients (9\%) showed lateral talar displacement in the weightbearing radiographs that were performed 3-10 days after the initial trauma. Then, the pain had mostly resolved, and the weightbearing was well tolerated by all patients. Further functional treatment was adapted to the patients' needs and discomfort (pain and swelling), and posed few problems. After a period of 18-120 months (mean 62) 47 of 51 patients $(92 \%)$ were questioned by phone and only four patients (9\%) reported minor ankle-hindfoot problems. Four patients had died, but had not had problems related to their ankle injury, according to their relatives.

The correlation of type of external support for nonoperative treatment of the stable SER-II ankle fractures (bandages, orthotics, softcast-boots, and below-knee cast) 
to functional outcome as measured by the AOFAS score was "poor" $(p \geq 0.05)$. In addition, the correlation of type of external support to complications (six delayed union) was "poor". Since we adapted the stability of the external support only to the severity of the patient's pain perception while the fractures were considered stable, no stronger correlation was expected in this study. In the literature, there is no evidence for superior or inferior external support of non-operative treatment of stable ankle fractures.

Risk factors and comorbidities, however, had a "moderate" impact on bone healing in the study group (correlation to delayed bone healing $r=0.58$ ). Present history of smoking within the last 5 years, diabetes and obesity have been previously identified as risk factors for bone healing, and were confirmed in this study [10].

There seems to be a group of patients with minor instability (partial deltoid ligament lesions or pre-existing laxity, or both) that is considered unstable on manual or gravity stress radiographs, but would appear stable on weightbearing radiographs. Schock et al. [9] found 55\% of their patients to be positive on gravity stress radiographs and recommended operation. On radiographs with manually applied stress McConnell et al. [6] reported 37\% unstable fractures in their 97 patients, and Egol et al. [14] 65\% in 101 patients. In contrast to these results, only $10 \%$ of our patients showed instability on weightbearing radiographs, and were recommended to have operative fixation. Since all our patients did well, this would suggest that most SER-II fractures are stable and that relevant instability is largely overestimated by the stress manual or gravity stress radiographs.

One of the possible explanations for this overestimation may be the fact that weightbearing increases the dorsiflexion of the talus, thus tightens the (remaining) deltoid fibers and pulls the talus medially. Although the manual and gravity stress radiographs are supposed to be performed with the ankle in a neutral dorsiflexion-plantarflexion position to be most predictive of deep deltoid ligament disruption it may be possible that many patients have their ankles in slight plantarflexion during the examination [15]. We have observed that in the early phase of healing of non-operatively treated fractures a side-toside play of the talus in the ankle mortise of about 1-2 mm was possible if the talus was plantarflexed. In dorsiflexion, this shift disappeared. After complete healing of the fracture, the instability was not reproducible anymore. Anatomic predispositions like varus-valgus hindfoot alignment and intrinsic stability of the joint surface (saddle shape) may similarly play a role.

Lauge-Hansen suggested that (stable) SER-II ankle fractures (approximately $30 \%$ of all ankle fractures) do not require anatomic reduction, and optimal function can be expected $[1,4]$. The long-term benign nature of SER-II ankle fractures has been proven [3]. Three prospective long-term outcome studies from Scandinavia reported on 213 SER-II fractures treated from 1950 to 1976 [3, 13, 16]. With non-operative treatment anatomic reduction was not always achieved after at mean follow-up of 17.9 years. However, similar percentages of patients/ankles treated non-operatively and operatively were symptom free: 148 of $178(83.1 \%)$ and 30 of $34(88.2 \%)$, respectively, and posttraumatic arthritis on follow-up radiographs occurred in a similar percentage of patients undergoing non-operative and operative treatment ( 5 of 178 , or $2.8 \%$, vs. one of 34 , or $2.9 \%$, respectively).

As opposed to stable SER-II ankle fractures, non-operative treatment of (unstable) SER-IV ankle fractures has been associated with inferior results [17]. The incidence of radiographic changes of posttraumatic arthritis in unstable SER ankle fractures treated non-operatively approached $50 \%$ at an average of 19 years' follow-up (range 3-34 years) $[3,13]$. Correlation of radiographic evidence of arthritis and clinical outcome was not clarified in these studies.

Although surgery for ankle fractures is considered a routine procedure, it carries a remarkable risk of complications (10.4\% in a recent literature review) [18]. Therefore, it is essential to accurately identify stable injuries and avoid unnecessary operations.

There are several limitations in this study. At latest follow-up, patients were interviewed by phone only, and no radiographs were taken. Therefore, asymptomatic ankle arthritis, ankle instability, or poor range of motion of the hindfoot joints might have been missed in some cases. However, since pain and function are the most important outcome parameters, we believe that even in the absence of radiographic follow-up conclusions about the clinical reliability of this test can be made. This is further substantiated by the study of Finnan et al. [19] who stated that the quality of reduction and the absence of arthritis correlated well to health-related quality of life. Long-term outcome studies have already proven the benign nature of these injuries [3].

In conclusion, performing weightbearing radiographs to distinguish unstable from stable isolated lateral malleolar fractures is an easy, pain-free, safe and reliable method to choose non-operative or operative treatment, with respect to clinical outcome. The delay of 3-10 days until the decision about surgical treatment is well accepted by the patients. Manual or gravity stress radiographs seem to overestimate the need for operation, since only $9 \%$ of our patients were advised to have operative fracture fixation.

\section{References}

1. Lauge-Hansen N, Ankelbrud I (1942) Genetisk diagnose og reposition (Fractures of the ankle, I: genetic diagnose and treatment). Dissertation. Munskgaard, Copenhagen 
2. Müller ME, Allgöwer M, Schneider R, Willenegger H (1991) Manual of internal fixation. Techniques recommended by the AO-ASIF group, 3rd edn. Springer, Berlin

3. Bauer M, Jonsson K, Nilsson B (1985) Thirty-year follow-up of ankle fractures. Acta Orthop Scand 56:103-106

4. Yde J, Kristensen KD (1980) Ankle fractures. Supination-eversion fractures stage II. Primary and late results of operative and non-operative treatment. Acta Orthop Scand 51(4):695-702

5. Egol KA, Amirtharajah M, Tejwani NC, Capla EL, Koval KJ (2004) Ankle stress test for predicting the need for surgical fixation of isolated fibular fractures. J Bone Joint Surg Am 86(11):2393-2398

6. McConnell T, Creevy W, Tornetta P 3rd (2004) Stress examination of supination external rotation-type fibular fractures. J Bone Joint Surg Am 86(10):2171-2178

7. Gill JB, Risko T, Raducan V, Grimes JS, Schutt RC Jr (2007) Comparison of manual and gravity stress radiographs for the evaluation of supination-external rotation fibular fractures. J Bone Joint Surg Am 89(5):994-999

8. Michelson JD, Varner KE, Checcone M (2001) Diagnosing deltoid injury in ankle fractures: the gravity stress view. Clin Orthop Rel Res 387:178-182

9. Schock HJ, Pinzur M, Manion L, Stover M (2007) The use of gravity or manual-stress radiographs in the assessment of supination-external rotation fractures of the ankle. J Bone Joint Surg Br 89(8):1055-1059

10. Easley M, Trnka H, Schon L, Myerson M (2000) Isolated subtalar arthrodesis. J Bone Joint Surg Am 82(5):613-624
11. Kitaoka HB, Alexander IJ, Adelaar RS, Nunley JA, Myerson MS, Sanders M (1994) Clinical rating systems for the ankle-hindfoot, midfoot, hallux, and lesser toes. Foot Ankle Int 15:349-353

12. Chan YH (2003) Correlational analysis (Biostatistics 104). Singap Med J 44:614-619

13. Yde J, Kristensen KD (1980) Ankle fractures: supination-eversion fractures of stage IV. Primary and late results of operative and non-operative treatment. Acta Orthop Scand 51(6):981-990

14. Egol KA, Tejwani NC, Walsh MG, Capla EL, Koval KJ (2006) Predictors of short-term functional outcome following ankle fracture surgery. J Bone Joint Surg Am 88(5):974-979

15. Park SS, Kubiak EN, Egol KA, Kummer F, Koval KJ (2006) Stress radiographs after ankle fracture: the effect of ankle position and deltoid ligament status on medial clear space measurements. J Orthop Trauma 20(1):11-18

16. Kristensen KD, Hansen T (1985) Closed treatment of ankle fractures: stage II supination-eversion fractures followed for 20 years. Acta Orthop Scand 56:107-109

17. Michelson JD, Magid D, McHale K (2007) Clinical utility of a stability based ankle fracture classification system. J Orthop Trauma 21:307-315

18. Gougoulias N, Khanna A, Sakellariou A, Maffulli N (2009) Supination-external rotation ankle fractures stability a key issue. Clin Orthop Relat Res (epub ahead of print)

19. Finnan R, Funk L, Pinzur MS, Rabin S, Lomasney L, Jukenelis D (2005) Health related quality of life in patients with supinationexternal rotation stage IV ankle fractures. Foot Ankle Int 26(12):1038-1041 